\title{
Shakespeares Hamlet und die Frauen: Rezeptionsgeschichte als Emanzipationsgeschichte
}

\author{
Ina Schabert (Universität München)
}

Wenn man die gängigen Geschichten der Shakespeare-Rezeption konsultiert, oder die Textausgaben zum Shakespeare-Criticism, so findet man für die ersten drei Jahrhunderte, bis ins frühe 20. Jahrhundert hinein, kaum Namen von Frauen. Bestenfalls die Autorin Madame de Staël, ferner die Mitübersetzerin der SchlegelTieck-Ausgabe, Dorothea Tieck, und einige Starschauspielerinnen werden erwähnt. Dies gilt auch für die Rezeptionsgeschichte in dem von mir herausgegebenen Shakespeare-Handbuch, denn wir wollten hier ja nicht unsere eigenen Positionen und Wertungen dokumentieren, sondern den allgemeinen Wissensstand.

Mein Vortrag bringt also eine Korrektur am Handbuchwissen an, eine Korrektur, die durch zahlreiche Arbeiten der Frauenforschung, vor allem des letzten Jahrzehnts, vorbereitet wurde und die überfällig ist. Denn Shakespeare ist für literarisch interessierte und ambitionierte Frauen seit dem 17. Jahrhundert wichtig gewesen, und umgekehrt haben Frauen dazu beigetragen, den Dramatiker im frühen 18. Jahrhundert gegenüber dem Klassizismus zu rehabilitieren, und ihn sodann aus immer neuen Blickwinkeln zu betrachten und lebendig zu halten. 


\section{Frauen solidarisieren sich mit Shakespeare}

Sehr früh schon hat Shakespeare, vermutlich ohne dies zu beabsichtigen, weibliches intellektuelles Selbstbewusstsein zu stärken vermocht. Ein neidischer Zeitgenosse, Ben Jonson, hatte ihm bescheinigt, er kenne kaum Latein und noch weniger Griechisch - „small Latin and less Greek “1 - und dieser sprichwörtlich gewordene (obgleich, wie die Forschung aufgezeigt hat, eher unberechtigte) Vorwurf mangelnder klassischer Bildung wurde für englische Frauen bald zum freudig genutzten Identifikationsmoment. Sie, die von formaler, systematischer Schulung, von public schools und Universitäten ausgeschlossen waren, erklärten Shakespeare zum Paradebeispiel eines Autors, der diese Voraussetzungen nicht nötig hatte, um großartige Werke zu schaffen. Nicht aus Büchern sondern aus dem eigenen Kopf, aus seiner Intuition und seiner Menschenkenntnis musste Shakespeare die Stoffe und die Gestaltungsideen für seine Dramen geholt haben. Im selben Sinn sahen sich nun auch Frauen zur Autorschaft berufen. Eine äußerst schreibfreudige Autorin und Shakespeare-Verehrerin, Margaret Cavendish, Herzogin von Newcastle, lässt sich um 1650 - abweichend von der ikonographischen Konvention, die einen Schriftsteller vor seinen Büchern zeigt - mit ihrer Schreibfeder vor einer leeren Wand malen, während Engel sie mit der Dichterkrone krönen.

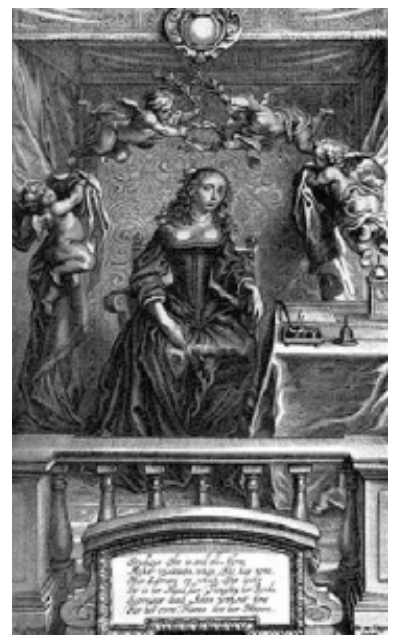

Margaret Cavendish, Frontispiz zu The Philosophical and Physical Opinions (London, 1655), Gravur von Peter van Schuppen nach einem Gemälde von Abraham van Diepenbeek.

\footnotetext{
${ }^{1}$ Die Formulierung stammt aus Ben Jonsons Nachrufgedicht auf Shakespeare in der ersten, postumen Gesamtausgabe seiner Werke: „And though thou hadst smalle Latine and lesse Greeke ... "(,To the Memory of my Beloved, The Author Mr. William Shakespeare“, zitiert nach The Norton Shakespeare, eds. Stephen Greenblatt u.a., New York: Norton 1997, S. 3351.
} 
Die einzige Quelle ihres Schreibens ist, so erfahren wir, Begabung und Erfahrung. Als Inschrift zu dem Porträt, das beim prominenten holländischen Maler Abraham van Diepenbeke in Auftrag gegeben wurde, erklärt ein kleines Gedicht diese Besonderheit:

Die Bibliothek, auf die sie setzt,

Das ist ihr Kopf, ihr Geist der Text.

Nicht tote Asche inspiriert

Die Frau, die eignes Feuer spürt.
Her Library in which She look's

It is her Head her Thoughts her Books,

Scorning dead Ashes without Fire

For her owne Flames doe her Inspire. ${ }^{2}$

In ähnlicher Weise begründet Aphra Behn, eine in den 1670er und 1680er Jahren sehr beliebte Dramatikerin, ihren Erfolg im Theater mit dem Präzedenzfall Shakespeare, der, wie sie hervorhebt, beim Publikum mehr Anklang fand als der gelehrte Jonson. Im Vorwort zu einem ihrer Werke, The Dutch Lover, schreibt sie: „Playes have no great room for that which is mens great advantage over women, that is Learning: We all well know that the immortal Shakespears Playes (who was not guilty of much more of this than often falls to womens share) have better pleas'd the World than Johnsons works. “3

Lange bevor in der Zeit der Frühromantik Treue zu „nature“ im Sinne einer intuitiv erfassbaren zeitlosen Natur des Menschen in der allgemeinen Literaturtheorie zu einem zentralen Wert avancierte, ist Shakespeare für Frauen der „poet of human nature", und als solcher das große Vorbild. Lewis Theobald, Herausgeber einer frühen Shakespeare-Ausgabe, vermerkt in seinem Vorwort 1726: „There is scarce a Poet, that our English tongue boasts of, who is more the Subject of the Ladies' Reading “. ${ }^{4}$ In den frühen 1730er Jahren wurde ein Shakespeare’s Ladies' Club gegründet, der sich dafür einsetzte, dass die bis dahin eher vernachlässigten und vielbemäkelten Dramen auf die Spielpläne kamen. Margaret Cavendish schreibt einen der ersten Shakespeare-Kommentare überhaupt, und auch im 18. Jahrhundert haben Frauen wie Charlotte Lennox, Elizabeth Montagu, Elizabeth Griffith und Elizabeth Inchbald in der Erschließung von Shakespeares Kunst eine Pionierrolle gespielt. ${ }^{5}$ Die Malerin Angelika Kauffmann ehrt den Dichter in den

\footnotetext{
${ }^{2}$ Der vollständige Text lautet: „Studious She is and all Alone, / Most visitants, when She has none. / Her Library in which She look's / It is her Head her Thoughts her Books. / Scorning dead Ashes without fire / For her owne Flames doe her Inspire."

${ }^{3}$ Aphra Behn, Vorwort zu The Dutch Lover, in: The Plays 1671-1677 [The Works, vol. 5], hg. Janet Todd, London: Pickering 1996, S. 162.

${ }^{4}$ Lewis Theobald, Shakespeare Restored, London 1726, S. vi.

${ }^{5}$ Siehe dazu Judith Hawley, „Shakespearean Sensibilities: Women Reading Shakespeare 1753-1808“, in Shakespearean Continuities: Essays in Honour of E.A.J. Honigmann, hg. Joan Batchelor u.a., Basingstoke: Macmillan 1997, S. 290-304.
} 
1770er Jahren mit einem Idealporträt und einer Vignette, in der die personifizierte Phantasie sein Grab schmückt.

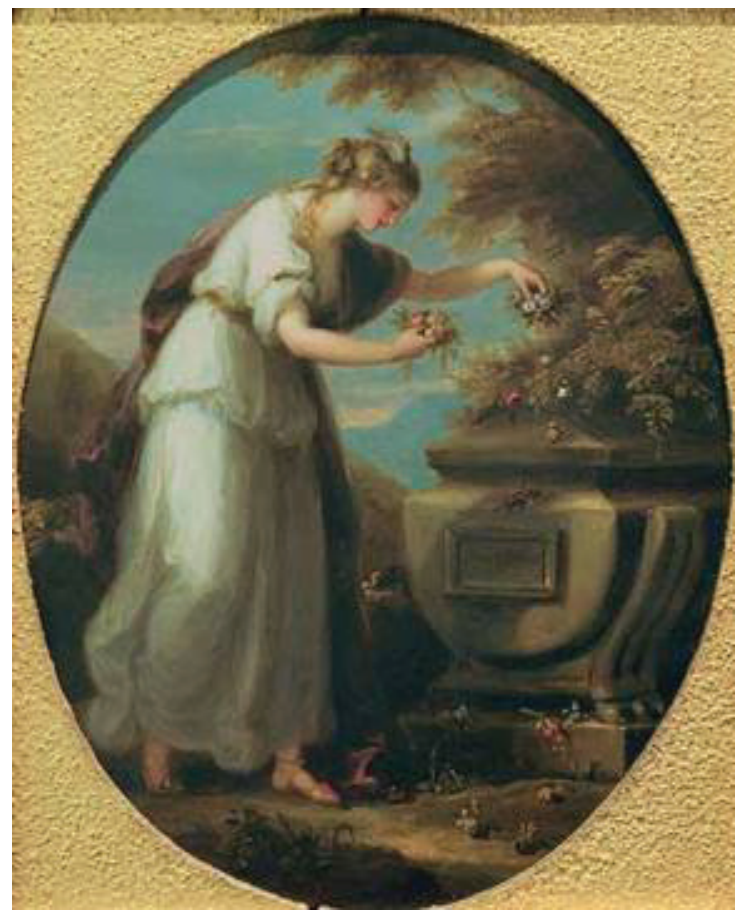

Angelika Kauffmann, Ausschnitt aus: „An Ideal Portrait of Shakespeare“ (1755)

Royal Shakespeare Company Collection (public domain).

Auch hier also, mit dem Bezug auf die Phantasie, der Hinweis auf Shakespeares autonome Schöpferkraft, und - durch deren weibliche Gestalt - die Andeutung einer speziellen Affinität zwischen den Frauen und Shakespeare. Besonders bemerkenswert in unserem Zusammenhang ist An Essay on the Writings and Genius of Shakespear, den Montagu 1769 veröffentlichte. Die Autorin, die sich als die elegante und geistreiche Gastgeberin des Salons der Bluestockings einen Namen gemacht hat, verteidigt in dieser Abhandlung den englischen Dramatiker temperamentvoll und mit manchmal satirischer Schärfe gegen die Angriffe des Franzosen Voltaire. Dieser habe Shakespeare mit klassizistischem Maß gemessen und ihn so fälschlich für mangelhaft befunden. Montagu hingegen erweist sich mit ihrem Shakespeareverständnis als dem großen Dramatiker kongenial, so stellt Mary Scott Taylor anlässlich ihrer Verteidigung weiblicher Autorschaft im Gedicht The 
Female Advocate (1774) fest. ${ }^{6}$ Weibliches intellektuelles Selbstbewusstsein verbindet sich in Montagus Schrift höchst wirkungsvoll mit kämpferischem Nationalstolz. Shakespeares kraftvolle englische Männlichkeit ermächtigt die Frau, die zu ihm hält und mit ihrer Feder für ihn kämpft.

\section{Autorinnen identifizieren sich mit Hamlet}

In der Zeit der Romantik entdecken auch schreibende Frauen in Frankreich Shakespeare als Mittel der Selbstaufwertung. In England ist der Dramatiker jetzt im Kanon und damit im männlichen Establishment angekommen; in Frankreich ist er der große Außenseiter, mit dem sich Künstler und Intellektuelle jenseits des Mainstream, und damit auch geistig tätige Frauen, solidarisieren. Germaine de Staël begreift und lobt in ihren europaweit beachteten Schriften De la Littérature (1800) und De l'Allemagne (1813) das Drama Hamlet als Inbegriff der düsteren, gedankenschweren Literatur des Nordens, d.h. Englands und Deutschlands, im Unterschied zur französischen Literatur. In ihrem Roman Corinne (1807) verliebt sich die autobiographisch konzipierte Titelfigur in einen hamlethaften britischen Lord. Der Lord und die idealisierte Selbstprojektion der Autorin in der Person von Corinne begegnen einander auf Augenhöhe: In moralischer und intellektueller Autorität wie auch im selbstbestimmten, unkonventionellen Verhalten ist de Staëls Heldin dem Helden Shakespeares mehr als gewachsen.

Wie hier, lässt sich ganz allgemein beobachten, dass ausgerechnet Hamlet, eine bis dahin in Frankreich eher verspottete oder ignorierte Figur, nun zum Objekt weiblicher identifikatorischer Aufmerksamkeit wird. Hamlet rückt neben die starken Frauen in den Dramen, die es den Leserinnen und Schauspielerinnen schon immer angetan hatten: Lady Macbeth, Cleopatra, Viola (in Twelfth Night / Was Ihr Wollt), die in Männerkleidern ihre eigene Zukunft bestimmt, die widerspenstige Katherine, die gezähmt werden muss, und Portia aus The Merchant of Venice. ${ }^{7}$ Erklärt wurden solch erstaunliche Charaktere damit, dass man im England der Königin Elizabeth I mächtige, selbständige Frauen zu schätzen gewusst habe. Die Schauspielerin Ellen Terry betrachtet in einer von Feministinnen gern zitierten Aussage die furchtlosen, energiegeladenen, resoluten und

\footnotetext{
${ }^{6}$ „Well hath thy Pen his [Shakespeare's] various beauties shown, / And prov'd thy soul congenial to his own“. Mary Scott Taylor, The Female Advocate, in: First Feminists: British Women Writers 15781799, ed. Moira Ferguson, Bloomington: Indiana University Press 1985, S. 363.

${ }^{7}$ Französische Autorinnen schenken daneben auch der Feenkönigin Titania aus A Midsummer Night's Dream, der rebellischen Mutterfigur Constance in King John und Portia, der mutigen Gattin des Brutus in Julius Caesar besondere Beachtung; siehe dazu Aimée Boutin, „Shakespeare, Women, and French Romanticism“, Modern Language Quarterly 65,4 (2004) 505-529.
} 
intelligenten Heldinnen der Dramen als Shakespeares „vindication of women“, d.h. als eine Frauenkampfschrift lange vor dem feministischen Pamphlet von Mary Wollstonecraft, auf dessen Titel (A Vindication of the Rights of Woman) sie anspielt. ${ }^{8}$ (Wollstonecraft selbst hat in ihren Schriften und Briefen gern auf Hamlet Bezug genommen; ihr Lieblingszitat war seine Klage, in 1. 2. 134, über die moralische Verderbtheit der Welt, die er mit einem ins Unkraut geschossenen Garten vergleicht - wobei sie allerdings den frauenfeindlichen Kontext dieser Klage ausblendet). Eleanor Marx ist, wie ihre Mutter, eine begeisterte ShakespeareLeserin und führt in ihrer mit ihrem Lebensgefährten Edward Aveling gemeinsam verfassten Streitschrift The Woman Question (1886) die starken Frauen in den Dramen als Vorbilder für die eigene Gegenwart an.

Shakespeares Ophelia hingegen, die für männliche Künstler des 19. Jahrhunderts eine Kultfigur war, ist für selbstbewusste weibliche Autoren und auch Schauspielerinnen eher ein Problem. Als Ellen Terry, die diese Rolle gegenüber Henry Irvings Hamlet zu spielen hatte, Ophelia ihrer Klage über den Tod des Vaters Polonius durch schwarze Trauerkleidung etwas mehr Eigenwert geben wollte, wurde sie barsch zur Ordnung gerufen. Sie musste auf das schon in Auftrag gegebene dunkle Kleid verzichten und, wie es seit jeher üblich war, in unschuldigem Weiß klagen und wahnsinnig werden:

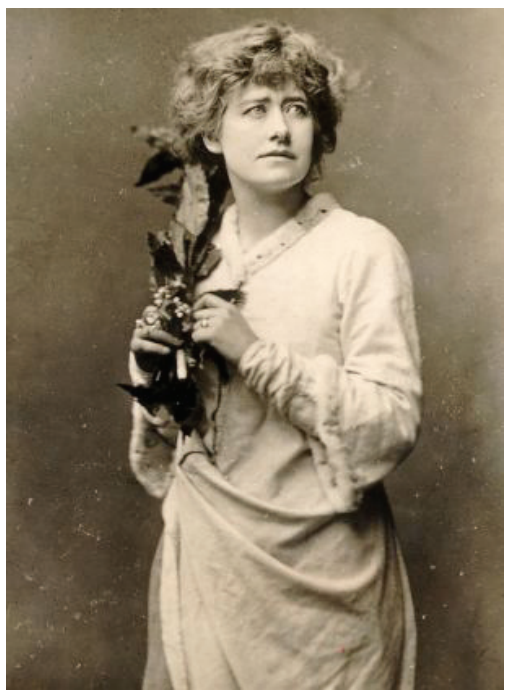

„Miss Ellen Terry as Ophelia in Shakespeare’s Hamlet“ (Photo ca. 1878)

Folger Shakespeare Library

${ }^{8}$ Ellen Terry, Four Lectures on Shakespeare (1911-1921), London: Martin Hopkinson 1932, S. 81. 
Seriöses Schwarz zu tragen, war Hamlets bzw. Irvings Privileg. ${ }^{9}$

Das Gefühl der Frauen, mit Hamlet verwandt zu sein, wurde gefördert und gerechtfertigt dadurch, dass die Romantik feminine Züge in Hamlet entdeckte. Vor allem Goethe war hier wegweisend mit der erzählten Lektüre und Aufführung des Hamlet in Wilhelm Meisters Lehrjahre (1795/96). Goethes Wilhelm betont Hamlets „schwankende Melancholie, seine weiche Trauer, seine tätige Unentschlossenheit“ und seine „zarte Seele“. ${ }^{10}$ Den „Schlüssel“ für die tragische Entwicklung des Stücks sieht Wilhelm darin, dass Hamlet aufgrund seiner zarten Konstitution dem ihm auferlegten Racheauftrag nicht gewachsen ist und deshalb zugrunde gehen muss:

Hier wird ein Eichbaum in ein köstliches Gefäß gepflanzt, das nur liebliche Blumen in seinen Schoß hätte aufnehmen sollen: die Wurzeln dehnen aus, das Gefäß wird zernichtet. ${ }^{11}$

Der Vergleich Hamlets mit einem ,köstlichen Gefäß', das zu fein und schwach für eine massive Belastung ist, nähert den Helden einer edlen Weiblichkeit an, die traditionell schweren Bürden nicht gewachsen ist; die Bildersprache der „lieblichen Blumen" und des "Schoßes“ verstärkt noch den Effekt der Femininität. Schon wenige Jahre später erklärt Sophie Mereau in ihrer Zeitschrift Kalasthiskos mit einem wortwörtlichen Zitat der Passage aus Goethes Wilhelm Meister diesen supersensiblen Hamlet zu ihrem eigenen. ${ }^{12}$ Auch Dorothea Schlegel entwirft um 1800 im Romanfragment Florentin einen femininen Helden in der Tradition von Goethes Hamlet. Die Münchener Malerin Marianne Kürzinger, die durch das heroische Staatsgemälde Gallia schützt Bavaria (1805) berühmt wurde, bekundet mit einer Serie von dreißig Bildern zu Hamlet (die leider verschollen sind) ihre Nähe zu dem Drama.

Der Maler Eugène Delacroix, ein Bewunderer Goethes, bestätigt dessen Hamlet-Lektüre bereits dadurch, dass er für seine Lithographien und Gemälde zu Hamlet als Modell für die Figur des Helden eine junge Frau, Marguerite Pierret,

\footnotetext{
${ }^{9}$ Ellen Terry, The Story of my Life, New York: Schocken 1982, S. 99-100.

${ }^{10}$ Goethe, Wilhelm Meisters Lehrjahre, Berlin, Weimar: Aufbau Verlag 1988 [Berliner Ausgabe, Bd. 10] S. 320 und 226.

${ }^{11}$ Ibid, S. 255

${ }^{12}$ Sophie Mereau, „Fragment eines Briefs über Wilhelm Meisters Lehrjahre“ (1799), abgedruckt in Mereaus Zeitschrift Kalasthiskos, 1. Bändchen, Berlin 1801, S. 233-234 (zum Teil nimmt die Forschung allerdings Clemens Brentano als Autor des Aufsatzes an).
} 
auswählt. Über das wohl berühmteste dieser Bilder, dasjenige, das Hamlet in der Totengräberszene, über dem ausgehobenen Grab für Ophelia, zeigt,

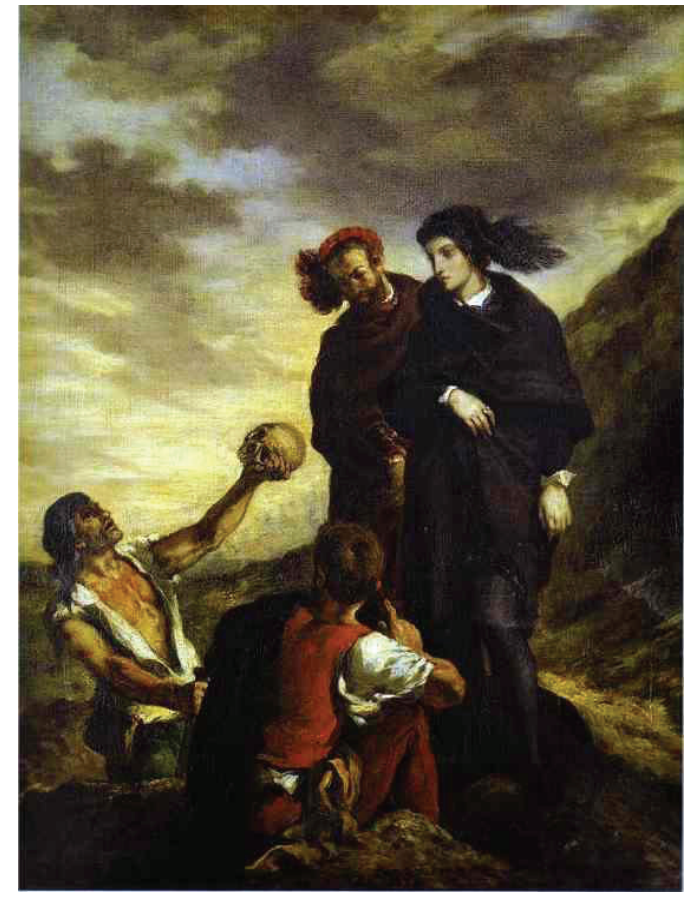

Eugène Delacroix, "Hamlet et Horatio au cimetière" (1839)

Paris : Musée du Louvre (public domain)

verfasst die Dichterin Louise Colet, die auch den Hamlet übersetzt hat, 1844 ein langes Gedicht. ${ }^{13}$ Delacroix, so befindet sie in diesem, malte mit dem empfindsamen Helden, der ebenso an seinem Genie wie an der Welt leidet, den idealen Hamlet, einen Hamlet, von dem Shakespeare, der an die Theaterrealität seiner Zeit gebunden war, nur träumen konnte: „Oh! C'est bien le Hamlet que Shakspeare rêva“. ${ }^{14}$ Colet beansprucht somit, einen unmittelbaren Einblick in Shakespeares Gedankenwelt zu haben. Frauen verlieben sich also nicht nur, wie Staëls alter ego

\footnotetext{
${ }^{13}$ Colet bezieht sich auf das frühere Bild von 1835, nicht auf das bekanntere Ölgemälde von 1839, das im Besitz des Louvre ist (Tony Howard, Women as Hamlet: Performance and Interpretation in Theatre, Film and Fiction, Cambridge: Cambridge University Press 2007, S. 12-13).

${ }^{14}$ „A M.*** sur son tableau de Hamlet“: „Seul tu nous a rendu le Hamlet de Shakspeare, / Création sublime où la douleur respire, / Où le poète a mis dans un cour dévasté / Tous les maux du génie et de l'humanité [...] Shakspeare avait laissé son âme dans Hamlet / Mais son œuvre n'était qu'un symbole incomplet, / Avant que tes pinceaux, trempés à son génie, / Eussent fait pour cette âme un corps en harmonie [...] / Oh! C'est bien le Hamlet que Shakspeare rêva; / L'enveloppe brisée où l'âme s'en va [...]“. Louise Colet, Poésies complètes, Paris: Charles Gosselin 1844, S. 129-131.
} 
Corinne, in hamletähnliche Männer, sie werden Hamlet gleich, vollziehen sein Seelendrama nach, identifizieren sich mit ihm. Autorinnen machen sich seine Worte zu eigen; sie schreiben, sagt Annette von Droste-Hülshoff selbstironisch, „wie ein transvestirter Hamlet". ${ }^{15}$

George Sand bezeugt bereits mit ihrem männlichen Pseudonym und ihrer Vorliebe für Männerkleider einen Hang, sich androgyn zu stilisieren. Androgyn, nicht einseitig männlich, wie sie betont. Die Formulierung eines Bekannten, Alexis Duteil, der befand, sie sei weder Mann noch Frau, sondern ein ,Mensch' (un être), scheint ihr sehr zu gefallen. ${ }^{16}$ So überrascht es nicht, dass sie eine besondere Nähe zu Shakespeares als feminin geltendem männlichen Helden bezeugt. In einem Essay über Hamlet (1845) nimmt sie an seinem Weltschmerz und seiner Verzweiflung teil, indem sie sich im intimen Dialog mit ihm imaginiert. Sie deutet sein Verhalten als den universalen "Schrei der Menschheit, die gegen sich selbst revoltiert“. „Seit Adam bis zu deinen Tagen, Hamlet, und bis zu unseren, ist die Stimme der Erde ein ewiges Schluchzen, das sich in der ewigen Stille der Himmel verliert ". ${ }^{17}$ George Sands Idol war der Schauspieler Philibert Rouvière, der einen ausgeprägt schwarz-melancholischen Hamlet spielte. Im Roman Consuelo (1842) entwirft Sand mit Albert von Rudolstadt einen dunklen Helden, den ein Schuldgefühl an die Grenze zum Wahnsinn bringt. Seine psychische Gefährdung wird, vergleichbar mit Wilhelm Meisters Hamlet-Diagnose, erläutert als ein Zustand, in dem eine machtvolle Imagination das Gehäuse des Hirns zu zerbrechen droht. ${ }^{18}$ Auch das alter ego eines weiblichen Hamlet gestaltet Sand, in ihrem autobiographisch getönten Roman Lélia (1833). Der Roman entsteht als Gegenstück zu der zwei Jahre zuvor entstandenen Komposition Lélio des mit Sand befreundeten Hector Berlioz. Blass und melancholisch tritt Sands Lélia bei einem Ball in der Hamlet-typischen dunklen Männertracht auf, tiefe Gedanken wälzend. Auch Frau ist Hamlet - so wieder die Botschaft. Mit einem späteren Roman, mit L'Homme de Neige (1859), löst sich Sand sodann von der Schwarzen Romantik und ihrer Hamlet-Konzeption. In einer innovativen Re-Lektüre von Shakespeares Stück

\footnotetext{
${ }^{15}$ Brief vom 5. Juli 1843 an Sibylle Mertens-Schaaffhausen (Briefe 1843-1848, hg. Winfried Woesler, Tübingen: Niemeyer 1992, S. 65)

16 „Prenez-moi donc pour un homme ou pour une femme, comme vous voudrez. Duteil dit que je ne suis ni l'un ni l'autre, mais que [je] suis un être", George Sand, Mai 1835 an Adolphe Guéroult (Correspondance, ed. G. Lubin, 25 Bd., Paris: Garnier 1964-1991, Bd. III, S. 327).

17 „Création sublime, n'est-ce donc pas que tu résumes en toi toutes les souffrances d'une âme pure jetée au milieu de la corruption et condamnée à lutter contre le mal qui l'étreint et la brise?“ - „ce cri de l'humanité contre elle-même [...] depuis Adam jusqu'à toi, Hamlet, depuis de tes jours jusqu'aux nôtres, la voix de la terre est un éternel sanglot qui se perd dans l'éternel silence des cieux." Essay „Hamlet", veröffentlicht im Almanach du Mois, Februar 1845.

http://www.gutenberg.org/files/28623/28623-h/28623-h.html (1.7.2014).

${ }^{18}$ George Sand, Consuelo, ed. Robert Sctrick, Paris: Phebus 1999, S. 253.
} 
präsentiert sie eine nicht mehr tragische, sondern eher pikareske, handlungsfreudige und in der Gegenwehr gegen den verbrecherischen Onkel erfolgreiche Hamletfigur als Protagonisten. Sein Erleben wird aus der Innenschau berichtet - ein Zeichen für die wiederum enge Verbindung zwischen ihm und der Erzählerin und Autorin. Das Theaterstück, das er, wie das Shakespearesche Vorbild, sich ausdenkt, um die Wahrheit über den Vatermord herauszufinden, nutzt Sand selbstbewusst, um ihr eigenes unzeitgemäßes Konzept einer antinaturalistischen Kunst zu illustrieren, die eine konstruktive Wahrheit vermittelt. ${ }^{19}$ Die Schriftstellerin Fanny Lewald, eine Vorkämpferin der Frauenrechtsbewegung, ermächtigt sich wiederum, indem sie die geistige Verwandtschaft mit Hamlet und seinem Schöpfer betont. Um 1880 pilgert sie zum Hamlet-Schloss Helsingør in Dänemark. Auf der Schlossterrasse (dort, wo einst der Geist von Hamlets Vater dem Sohn den Racheauftrag erteilte) fühlt sie sich eins mit Shakespeare und seinem Helden, der „in jedem hochgestimmten Herzen" "große Gedanken, großes Empfinden“ erweckt. „Hier“, so ruft sie aus, „habe ich sie wieder einmal in ihrer ganzen Göttlichkeit und Unsterblichkeit empfunden, die Kraft der Dichtung. ${ }^{\text {"20 }}$

Die englische Autorin George Eliot (die nach Sands Vorbild wiederum unter einem männlichen Pseudonym schreibt), entwirft im Roman Daniel Deronda (1876) mit dem Titelhelden ihren Hamlet. Etwa gleichzeitig stellt sie sich in einem längeren philosophischen Gedicht vor, wie sich ein in ihre eigene Gegenwart versetzter Hamlet in einem Oxforder College mit den Kommilitonen Horatio, Laertes, Osric, Rosencrantz und Guidenstern und einem Studentenpfarrer zu einem Streitgespräch trifft. ${ }^{21}$ Hamlets Vorgeschichte als Student in Wittenberg gewinnt jetzt, in den 1870er Jahren, als Frauen für das Recht auf ein Universitätsstudium kämpfen, für die weibliche Hamletrezeption besondere Bedeutung. Eliot stellt die jungen Männer dar in einer vielseitigen, anspruchsvollen und aktuell informierten Diskussion und führt damit den Beweis, dass auch sie, als Autorin des Texts, zur intellektuellen Avantgarde ihrer Zeit gehört und zweifellos die Reife für ein Universitätsstudium gehabt haben würde. Hamlets vielkommentiertes Zögern, seine Unschlüssigkeit, ist aus der Shakespeareschen Handlung in den viktorianischen Ideenkontext transponiert worden; er steht unentschieden zwischen christlichem Glauben und Naturwissenschaft, dichterischem Idealismus und rational argumentierender Philosophie.

\footnotetext{
${ }^{19}$ Siehe dazu Ina Schabert, „,The Play's the Thing: George Sand's Hamlet-Novel L'Homme de Neige“, Poetica 45, 3-4 (2013) 333-346.

${ }^{20}$ Fanny Lewald, Vom Sund zum Posilip: Briefe aus den Jahren 1879 bis 1881, Salzwasser Verlag: Paderborn 2013, S. 75-76.

${ }^{21}$ George Eliot, „A College Break-Fast Party“ (1874). Abdruck in Poems 1908, S. 416-440 www.unz.org/Pub/EliotGeorge-1908v09A-00001 (1.7.2014).
} 
Auch Eliots Kollegin, die Romanautorin und Journalistin Margaret Oliphant, sieht anlässlich einer Besprechung von Henry Irvings Hamlet-Aufführung (1878) Shakespeares Helden gekennzeichnet von der intellektuellen Krise ihrer eigenen Zeit. Eliot begegnet ihrem von Zweifeln geplagten Hamlet mit einem Hauch von Ironie, die vielleicht auch Selbstironie ist, Oliphant hingegen ist voller Sympathie für diese Figur, die sie umschreibt als:

the strange and wonderful being whom we never completely understand [...] but whom, at least, we know, as we know few other of our life-long friends. [Hamlet] is part of our intellectual training, [...] that impersonation of all the doubts and questionings of humanity.

Wieder geht es um humanity, die Menschheit, nicht um Frauen oder Männer. ${ }^{22}$ Auch Oliphant beansprucht mit ihrer Formulierung nicht Hamlets männliches Ich, sondern eine gemeinsame Humanität von Männern und Frauen, welche die Geschlechterdifferenz transzendiert. Hamlet ist einfach ein Mensch, „Hamlet was human", befindet in diesem egalitären Sinn auch noch die finnische Schauspielerin Leea Klemola, als sie 1995 den Hamlet spielt. ${ }^{23}$

\section{Schauspielerinnen spielen den Hamlet}

Indem sie selbst den Hamlet auf der Bühne verkörpern, können Frauen den Emanzipationseffekt optimieren, der aus der Identifikation mit dieser Figur resultiert. Der Beruf der Schauspielerin war im 19. Jahrhundert einer der wenigen angesehenen Frauenberufe überhaupt. Mehr noch wie die Schriftstellerin konnte die Schauspielerin durch ihre Tätigkeit berühmt und reich, d.h. materiell unabhängig, werden. So erklärt es sich, dass erfolgreiche Schauspielerinnen einen Stoßtrupp in der Frauenbewegung des späteren 19. Jahrhunderts bildeten. 1908 institutionalisierten sie sich in England in einem Kampfbund für das Frauenwahlrecht, in der Actresses' Franchise League, parallel zum Bund der Schriftstellerinnen, der Women Writers' Suffrage League.

\footnotetext{
22 ,the strange and wonderful being whom we never completely understand [...] but whom, at least, we know, as we know few other of our life-long friends. [Hamlet] is part of our intellectual training [...] that impersonation of all the doubts and questionings of humanity" (Review Essay of 1878, repr. in Critical Responses to Hamlet 1600-1900, eds. David Farley-Hills u.a., Bd. IV, AMS Press 2006, Teil II, S. 474-500: Zitat S. 474-75).

${ }^{23}$ Zitiert nach Howard, Women as Hamlet, S. 26.
} 
Die Aneignung der Rolle des Hamlet steht in Zusammenhang mit dieser Entwicklung. Zwar gab es seit dem früheren 18. Jahrhundert immer wieder weibliche Hamlets auf der Bühne, dies jedoch vornehmlich aus dem pragmatischen Grund, dass in einer Truppe gerade kein geeigneter männlicher Schauspieler zur Verfügung stand. Die in England und Frankreich populäre Konvention des travestiSpiels mit seinen erotischen Reizen - nur hier konnten Frauen in engen Männerstrumpfhosen Bein zeigen - hat solches Cross-casting begünstigt. Aufgrund der Hosenrollen in den Komödien waren die Frauen ohnehin darin geübt, in Shakespeares Stücken den Mann zu spielen und das Publikum war das gewöhnt. Die Hamlet-Rolle ist für ambitionierte Schauspielerinnen besonders attraktiv; mit ihrer breiten Palette von Spielnuancen geht sie weit über die Möglichkeiten weiblicher Rollenfächer hinaus. So nehmen die weiblichen Hamlets um $1850 \mathrm{zu}$, als zunehmend mehr Frauen die leitende Funktion eines actor manager bekommen und Programm und Besetzung selbst bestimmen.

Eine Spur feministischer Aufsässigkeit ist bei solchem Theater-Transvestismus nie ganz auszuschließen. Das Cross-casting macht mit dem weiblichen Schauspieler in männlicher Rolle unvermeidlich aufmerksam auf den Unterschied zwischen biologischem Geschlecht und kulturell vorgegebenem Geschlechtscharakter, zwischen sex und gender. Es widerlegt die Annahme, dass die traditionellen Verbindungen von beidem naturgemäß und unauflösbar sind und stellt damit in Frage, dass Frauen feminin sein müssen.

Im späteren 19. Jahrhundert nun nutzen Frauen das geschlechtswechselnde Spiel und vor allem die Hamlet-Rolle ganz gezielt zur emanzipatorischen Manifestation. In solchen Inszenierungen wird (wie in George Eliots Gedicht) dem Hinweis besonderes Gewicht gegeben, dass Prinz Hamlet im zu Beginn des im Drama dargestellten Geschehens sein Studium in Wittenberg unterbrochen hat, um an den dänischen Königshof zurückzukehren. Um die studentische Identität hervorzuheben, tritt die Schauspielerin als Hamlet gern in einem akademischen Talar auf. Die Frau als Student verkörpert ein Wunschbild von Mädchen und Frauen, die nur äußerst zögerlich zum Studium oder gar zu akademischen Berufen zugelassen wurden. Eine frauenemanzipatorische Bedeutung bekommt zudem die Fechtszene zwischen Hamlet und Laertes im letzten Akt, in der Hamlet der geschicktere ist. Das sportliche Können, das hier von einem weiblichen gegenüber einem männlichen Schauspieler demonstriert wird, unterstreicht die seit der Mitte des 19. Jahrhunderts aktuelle Forderung, dass auch Mädchen das Recht und die Befähigung zum Sport und zum Sportunterricht haben. Dem Fechtsport kam in 
dieser Debatte besondere Bedeutung zu, da das Fechten mehr Geschicklichkeit als Kraft erfordert. ${ }^{24}$

Nur einige der weiblichen Hamlets, bei denen die Doppelheit von Schauspielkunst und emanzipatorischem Bestreben offenkundig ist, können hier genannt werden. ${ }^{25}$ In keinem Fall handelt es sich dabei um den Versuch einer Frau, sich als wirklicher Mann auszugeben. Was dem Publikum geboten wird, ist vielmehr eine Frau, oft ein von weiblichen Rollen her bekannter und beliebter Star, der einen Mann spielt. In diesem Spiel werden weibliche und männliche GenderZuschreibungen miteinander vermischt, ineinander verquickt. Suggeriert wird ein distanzierter, flexibler, von Ironie nicht ganz freier Umgang mit solchen Zuschreibungen.

Besonders beeindruckend war der Hamlet der Felicita(s) von Vestvali, einer Schauspielerin deutscher Herkunft. Als Anna Marie Stägemann 1829 in Stettin geboren, riss sie als 15jährige, als Junge verkleidet, von zuhause aus. Sie machte erst in Paris, dann in den USA eine Karriere als Opernsängerin, später als Schauspielerin. Ihr Hamlet wurde 1866 in Amerika und England allgemein bewundert; das blonde Haar der Vestvali, die männlich hochgewachsene Figur und die sonore AltStimme waren, wie die Kritik feststellte, für den androgynen Charakter der Rolle ideal.

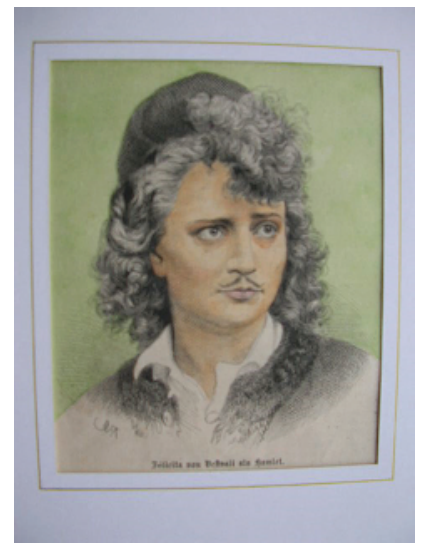

Felicitas von Vestvali als Hamlet, teilkolorierter Holzstich von 1873 (zvab.com)

Sie selbst identifizierte sich so sehr mit damit, dass sie sich gern ,Hamlet' nannte. 1868 wagte sie mit dem Stück eine Gastspielreise in deutsche Theater, die ein

\footnotetext{
${ }^{24}$ So die Argumentation im Beitrag von Mary Dane und Mary Howarth, „Lady Fencers: How Ladies Can Be Graceful and Athletic", The Harmsworth Magazine, July 1899.

${ }^{25}$ Eine ausführliche Darstellung bietet die Monographie von Howard, Women as Hamlet.
} 
unerwartet großer Erfolg wurde - unerwartet aufgrund des zu Recht befürchteten besonderen Vorurteils der Deutschen gegen solchen Transvestismus. Das Publikum war sehr skeptisch, berichtet der Leipziger Kritiker Rudolf von Gottschall; doch, so fährt er fort, diese Skepsis verflog mit ihrem ersten Auftritt:

Vergessen war vor der Macht des Genies alles, was man vorher, von den verschiedenartigsten Standpunkten aus gegen das Männerrollenspiel einer Frau hatte deutlich machen wollen; der Eindruck, den dieser Hamlet hervorbrachte, war ein gewaltiger. ${ }^{26}$

Im Jahr 1882 dokumentiert sich die Verknüpfung von Hamlet-Rolle und Frauenrechtsbewegung in der Amerikanerin Anna Dickinson (1842-1932). Im Bürgerkrieg war die junge Quäkerin eine mutige Kämpferin für die Rassengleichheit, was ihr den Ehrentitel einer ,Johanna von Orleans der Unionisten' einbrachte. 1864 hielt sie als erste Frau eine Rede vor dem amerikanischen Kongress. Nach dem Bürgerkrieg macht sie die Gleichberechtigung der Geschlechter zu ihrem Anliegen und hält in verschiedensten amerikanischen Städten Vorträge zur Lage der Frau. In ihrem Buch A Ragged Register sagt sie für die nahe Zukunft eine Angleichung der Geschlechter voraus. Gleichzeitig arbeitet sie als Schauspielerin und tritt 1882 am New Yorker Broadway in der Rolle des Hamlet auf. Es scheint, dass der knabenhafte, stille, eher rezitative Hamlet der schlanken kleinen Frau an sich nicht besonders beeindruckend war. Doch aufgrund ihrer politischen Berühmtheit war er ein vielbeachtetes Statement. Auch die Frauenrechtlerin Janette Steer spielt in einer von ihr selbst zusammengeholten Schauspielertruppe 1899 in Birmingham und London erfolgreich den Hamlet. Sie war ein frühes und sehr aktives Mitglied der Actresses' Franchise League, und musste 1912 wegen einer Protestaktion sogar eine kurze Haftstrafe verbüßen.

Die Jahrhundertwende brachte einen weiteren Höhepunkt in der Einvernahme der Hamlet-Rolle durch die Frauen. Eine Sensation bei der Pariser Weltausstellung 1900 war eine verfilmte Szene aus der Hamlet-Inszenierung von und mit Sarah Bernhardt.

\footnotetext{
${ }^{26}$ Rosa von Braunschweig, „Felicita von Vestvali“, in: Magnus Hirschfeld (Hg.), Jahrbuch für sexuelle Zwischenstufen (1903) http://www.omofonie.it/genn2006/vestvali.pdf S. 437.
} 


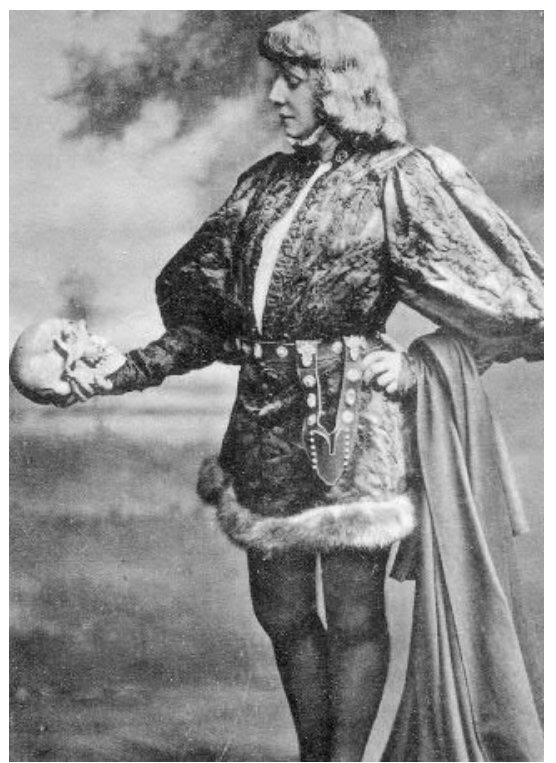

Sarah Bernhardt als Hamlet in La tragique histoire d'Hamlet. Théâtre Sarah Bernhardt, Paris 1899 (Postkarte des Deutschen Theatermuseums München)

Bernhardt war eine sehr selbstbestimmte Frau. Sie war eine Unternehmerin, die über eine eigene Theatertruppe verfügte und eines der großen Theaterhäuser im Zentrum von Paris gepachtet hatte, das sie in ,Théâtre Sarah Bernhardt' umbenannte. Für ihren Hamlet gab sie eine eigene Übersetzung (bei Marcel Schwob und Eugène Morand) in Auftrag, in Prosa statt der bisher üblichen gereimten Alexandriner. Der neue Text war ungewöhnlich vollständig - fünf Stunden dauerte die Aufführung. Bernhardt war der Auffassung, nur Frauen könnten den Hamlet überzeugend spielen: jüngere Männer hätten nicht die ausreichende intellektualisierte Sensibilität; später seien sie zu alt für die Rolle. Sie selbst gibt einen für die Zeit neuartigen, couragierten Hamlet. Hamlet ist für sie ein tatkräftiger Rächer, und nicht mehr die zarte Seele der romantischen Interpretationen des 19. Jahrhunderts. Einer Londoner Zeitung erklärte sie:

What people are determined to see in Hamlet is a feminized, hesitating, bemused creature. What I see, however, is a person who is manly and resolute, but nonetheless thoughtful. As soon as Hamlet encounters the spirit of his father and grasps the nature of the crime, he decides to avenge him. But since he is the opposite of Othello, who acts 
before he thinks, Hamlet characteristically thinks before he acts, a trait indicative of great strength and great spiritual power. ${ }^{27}$

Szenen, die lange ignoriert worden waren, weil sie in das feminin verfeinerte Hamletbild nicht passen, werden von ihr wieder aufgenommen, wie z.B. die Gebetszene mit der zynischen Überlegung des Helden, Claudius nicht beim Beten zu töten, weil er dann als reuiger Sünder in den Himmel kommen würde, oder die brutale Geste, mit der er verächtlich die Leiche des von ihm wie eine Ratte getöteten Polonius von der Bühne schleift. Auch Hamlets schroffe Haltung gegenüber Ophelia und seine Impertinenz im Umgang mit Polonius, die vordem abgemildert wurden, werden von Bernhardt voll ausgespielt. Gastspiele in den europäischen Metropolen und in den USA machten ihren Hamlet weltberühmt. Der kurze Stummfilm für die Weltausstellung zeigt kennzeichnenderweise die Fechtszene, in der Hamlet den Laertes, bzw. Sarah Bernhardt den Schauspieler Pierre Magnier besiegt. Sie wird als Duellritual nach den strengen Regeln der Fechtkunst ausgespielt. ${ }^{28}$

Die Bewertung der Aufführung - u.a. in Paris, New York, London und Stratford - reichte von absoluter Bewunderung über die höfliche Anerkennung einer ungewöhnlichen Schauspielkunst bis zur totalen Ablehnung. Besonders massive Kritik äußert der Berichterstatter des Jahrbuchs der Deutschen Shakespeare Gesellschaft, der Journalist und Reisebuchautor Eugen Zabel, der in seiner „gerechten Empörung“ über den von einer Frau gespielten Hamlet auch die Autorität des Präsidenten der Gesellschaft hinter sich weiß. In seinem naivrealistischen Verständnis, welches das Theater als „Abbild des unmittelbaren Lebens" auffasst, müssen Hamlet-Darsteller männlichen Geschlechts und, wie er sagt, ,jeder Zoll ein Mann“ sein. Bernhardts Hamlet ist für ihn der Tiefpunkt einer langen perversen Entwicklung, die im 18. Jahrhundert mit dem Bühnenstar Sarah Siddons begonnen hatte:

Von Sarah Siddons zieht sich die lange und unheilvolle Kette bis zu einer noch lebenden Sarah, Madame Bernhard (sic), die dieses Experiment nicht nur in Paris, Wien und London ausgeführt, sondern es auch fertiggebracht hat, an der Geburtsstätte des großen Briten, in Stratford on Avon, in dieser Rolle aufzutreten.

\footnotetext{
${ }^{27}$ Bernhardt in einem Brief an den Daily Telegraph vom 17. Juni 1899, zitiert bei Gerda Taranow, The Bernhardt Hamlet: Culture and Context, New York: Peter Lang 1996, S. 68.

${ }^{28}$ Ausschnitthafte Wiedergabe in http://www.youtube.com/watch?v=Mp_v_dP8s-8 (3. 4. 2014).
} 
„Die Hüter dieses Heiligtums“ so befindet er, haben damit „eine schwere Schuld auf sich geladen“. Weibliche Hamlets sind für Zabel schlicht ein „Unkraut“, ein „Übel, das bekämpft und ausgerottet werden muß“. Frauenfeindlichkeit und Frankreichfeindlichkeit vermischen sich in dem Bericht mit antisemitischen Untertönen. ${ }^{29}$

Im ersten Jahrzehnt des neuen Jahrhunderts, in der großen Zeit der Suffragettenbewegung, wird der Schluss-Szene von Hamlet eine einschlägige Aussage für das Frauenwahlrecht abgewonnen. Hamlet gibt (in einer Zeile, die ansonsten meistens gestrichen wurde) unmittelbar vor seinem Tod seine Stimme ab für den Norweger Fortinbras als neuem König - Shakespeare nimmt für Dänemark offenbar ein Wahlkönigtum an. Wenn eine Schauspielerin jetzt diese Worte spricht: „he has my dying voice", so darf wenigstens auf der Bühne und in einer Männerrolle eine Frau einmal wählen! Und ihre Stimme gibt sogar den Ausschlag. Allerdings werden in diesen Jahren die weiblichen Hamlets seltener. Das sich entwickelnde männlich dominierte Regietheater findet wenig Geschmack an solcher Art weiblichem Starkult. Die Feministinnen ihrerseits setzen auf die direkteren Botschaften der Ibsenstücke, der New Women Plays und des Straßentheaters der Suffragetten.

1914 jedoch, als die im Krieg befindlichen jungen Männer ersetzt werden müssen, erlebt die Transvestie auf der Bühne unerwartet eine neue Blüte. Wie in der Lebenswelt, so übernehmen jetzt Frauen im Theater in großem Ausmaß Männer-Rollen. Nicht nur die männlichen Hauptrollen werden mit weiblichen Stars besetzt; manche Inszenierungen arbeiten fast ausschließlich mit weiblichem Personal. Lilian Baylis, die den Betrieb des Old Vic-Theaters in London mit Schauspielerinnen aufrecht hält, schärft diesen ein: Es darf auf der Bühne kein Geschlecht geben - „There should be no sex in acting“". ${ }^{30}$ Der biologische Unterschied ist, wenn Theater gespielt wird, nicht von Bedeutung; das kulturelle Geschlecht hingegen, Gender, wird performativ erzeugt.

${ }^{29}$ Eugen Zabel, „Weibliche Hamlets“, Jahrbuch der Deutschen Shakespeare-Gesellschaft 36 (1900) 249-255. Ähnlich beklagte auch William D. Howells die dreifache Impertinenz von Bernhardts weiblichem, französischem und jüdischem Hamlet („A She-Hamlet“, in Literature and Life, New York: Harper and Brothers 1902).

${ }^{30}$ Sibyl and Russell Thorndike, Lilian Baylis, London: Chapman and Hall 1938, S. 57-58. Zit. nach Howard, Women as Hamlet, S. 119. 


\section{Die geschlechterideologische Bedeutung des Frauentheaters}

Im Frauentheater des Ersten Weltkriegs lässt sich eine Ironie der Geschichte sehen. Auf den Bühnen der Shakespearezeit traten, wie man weiß, nur männliche Schauspieler auf - die jungen Mädchen wurden durch Knaben vor dem Stimmbruch, die reiferen Frauen durch ältere Männer dargestellt. Praktisch möglich war dies durch das hervorragende Training der Akteure und eine vom äußeren Erscheinungsbild abstrahierende Kompetenz der Zuschauer. Gerechtfertigt wurde es durch ein Geschlechterkonzept, welches dem Mann als dem höchsten irdischen Glied in der Schöpfung alle Kompetenzen der unter ihm rangierenden Geschöpfe und damit auch der Frau zusprach. Umgekehrt definierte sich Weiblichkeit durch den Mangel an männlich-menschlicher Vollkommenheit. Folglich konnte der Mann alle Rollen spielen; ein Frauentheater aber wäre ein Ding der Unmöglichkeit gewesen, denn der Frau stehen eben nicht alle Fähigkeiten des anderen Geschlechts zur Verfügung. Diese frühneuzeitliche Vorstellung von der Hierarchie des Seins rückt später zugunsten einer kontrastiven Interpretation der Geschlechterdifferenz (two-sex model) in den Hintergrund. Entgrenzungsphänomene wie der Theatertransvestismus werden von dieser binären Geschlechterideologie mit Argwohn betrachtet.

Dennoch wird die frühere Argumentation gelegentlich wieder aufgenommen. Goethe bewundert in einer Abhandlung über „Frauenrollen auf dem römischen Theater durch Männer gespielt" (1788) das männliche Transvestitentheater, das er kurz zuvor in Rom gesehen hat. Männer beobachten Frauen, so befindet er; sie reflektieren über das, was sie beobachten, und sie gestalten ihr Spiel auf der Bühne als Resultat ihrer Überlegungen. Sie verwandeln damit weibliche Lebenswelt in Kunst. Frauen hingegen könnten im Grunde überhaupt nicht Theater im Sinne von Theaterkunst machen; sie spielten lediglich immer nur sich selbst. ${ }^{31}$ Mit ähnlicher Begründung spricht Max Behrboom um 1900, im Blick auf die weiblichen Hamlets, den Frauen die Kompetenz für das Theater ab: „A man contains in himself the whole of a woman's nature plus certain other qualities which make the difference between him and her“. [...] „Creative power, the power to conceive ideas and execute them, is an attribute of virility; women are denied it “. 32 Dass nun Regisseurinnen und Schauspielerinnen ganze Inszenierungen

\footnotetext{
${ }^{31}$ Goethe, „Frauenrollen auf dem Römischen Theater durch Männer gespielt“, Schriften zur Literatur, ed. Ernst Beutler, Zürich: Artemis 1949 [Gedenkausgabe der Werke, Briefe und Gespräche, Bd. 14], S. 9-13.

${ }^{32}$ Max Behrboom, More Theatres: 1898-1903, London: Rupert Hart-Davis, 1969, p. 37 (zitiert nach Howard, Women as Hamlet, S. 113). Zur Geschichte des Vorurteils siehe Karl Scheffler, Die Frau und die Kunst, Berlin: Julius Bard 1908.
} 
bestreiten und diese von hohem künstlerischem Wert sein können, ist vor solchem Hintergrund eine geschlechterideologische Sensation.

Im Verlauf des Kriegs artikulieren sich allerdings zunehmend Verdrängungsängste. Die Schauspielerinnen, so wird betont, dürfen den hoffentlich bald heimkehrenden Soldaten nicht die Berufschancen im Theater nehmen. Dennoch waren, nachdem die Frauen so vielseitig ihren Mann gestanden hatten, auch die ersten Nachkriegsjahre durch eine relativ frauenfreundliche Atmosphäre gekennzeichnet. 1921 wurde noch einmal ein vielbeachteter, in der Titelfigur weiblich besetzter Hamlet mit stark emanzipatorischem Akzent aufgeführt, und zwar der zu Recht berühmt gewordene Berliner Stummfilm von Asta Nielsen und Svend Gade. Wieder bestimmt hier eine Schauspielerin die Produktion mit, wenngleich nicht so absolut wie im Fall der Sarah Bernhardt. Es handelt sich nicht um eine Verfilmung des Shakespeare-Textes, sondern eine eigenständige Neufassung des Stoffs von Edward P. Vining. Dieser amerikanische Hamlet-Fan hatte 1881, offensichtlich inspiriert von den femininen Hamlet-Deutungen und der Welle weiblicher Hamlets im Theater, die These vertreten, der Held sei in Wirklichkeit eine Frau gewesen (es fragt sich, was mit dieser, Wirklichkeit' gemeint war - wohl kaum die der rauhen germanischen Sagenwelt von Shakespeares Quelle). Hamlet sei als Mädchen geboren und, nachdem es das einzige Kind von Gertrude war, von ihr als Knabe erzogen worden, um die Thronfolge innerhalb der Familie zu sichern. ${ }^{33}$ Vinings Buch wurde, wie es mit Spekulationen dieser Art häufiger geschieht, weithin bekannt. Sigmund Freud hat darauf Bezug genommen; in James Joyces Ulysses erinnert sich Leopold Bloom an das Buch, als er 1904 bei seinem Gang durch Dublin auf ein Poster mit der Schauspielerin Millicent Bandman-Palmer als Hamlet stößt ${ }^{34}$ Mit der hier eingeführten Erzählfigur des Mädchens, das mit Erfolg - ohne dass die Umwelt dies ahnt - ein Leben als junger Mann führt, wird der Geschlechterrollenwechsel aus dem Theaterspiel in die fiktionale Realität verlagert. Damit wird die Unabhängigkeit kultureller Geschlechtsmerkmale vom biologischen Geschlecht besonders überzeugend belegt. Autorinnen hatten das Motiv bereits zuvor in dieser Absicht eingesetzt: die Schauspielerin und Schriftstellerin Mary Robinson nutzte es im Roman Walsingham (1797) und George Sand in der dramatischen Erzählung Gabriel (1839).

Vinings Hamlet-Phantasie wird also zur Grundlage des Drehbuchs für den Hamlet-Film - vermutlich auf Anregung von Asta Nielsen, denn Svend Gade fand

\footnotetext{
${ }^{33}$ Edward P. Vining, The Mystery of Hamlet: An Attempt to Solve an Old Problem, Philadelphia: J.B. Lippincott and Co. 1881.

${ }^{34}$ James Joyce, Ulysses, Harmondsworth: Penguin 1968, S. 77.
} 
Vinings Idee bizarr. ${ }^{35}$ Nielsen mit ihrer schlanken, androgynen Figur erwies sich als die ideale Besetzung der Titelrolle.

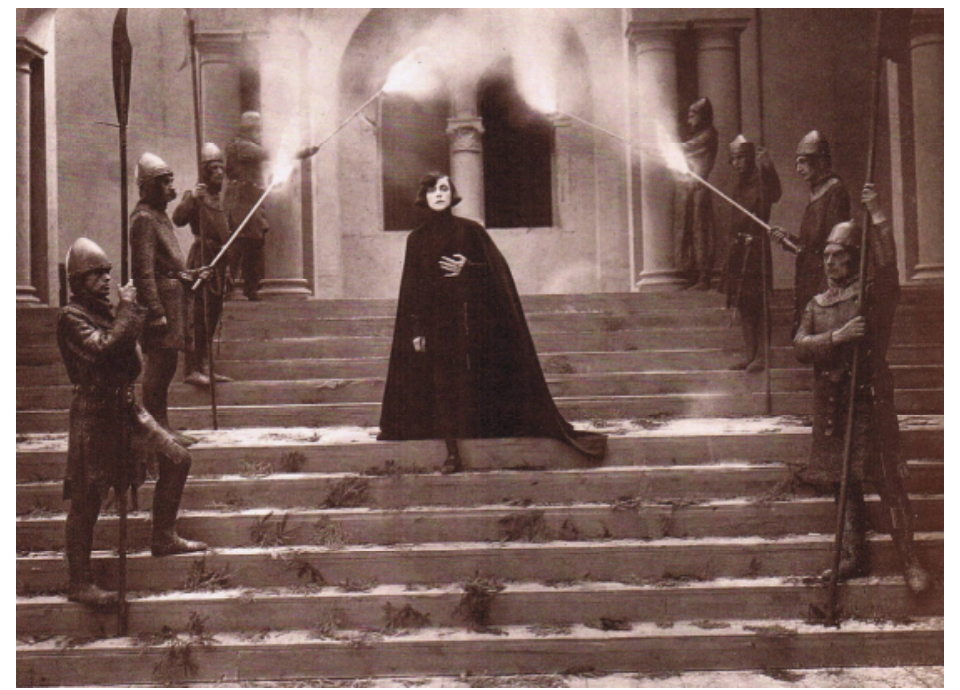

Asta Nielssen as Hamlet in Hamlet: A Drama of Vengeance (1920/21) film still (http:/blogs.flinders.edu.au/flinders-news/tag/hamlet-drama-of-vengeance)

Hamlet, Horatio, Laertes und Fortinbras sind in diesem Film Kommilitonen an der Wittenberger Universität - das Mädchenstudium ist noch immer aktuelles Thema. In Vorwegnahme der Fechtszene im letzten Akt von Shakespeares Stück wird früh eine Sequenz eingefügt, in der die vier Freunde Fechtübungen machen, und Hamlet sich durch besondere Geschicklichkeit auszeichnet. Dem Medium gemäß wird somit in der Stummfilmversion von Hamlets Studentenleben George Eliots Ideenstreit durch eine visuell vermittelbare typisch männliche Beschäftigung ersetzt. Neu ist die Betonung einer kameradschaftlichen Beziehung zwischen den jungen Männern und einem Hamlet, von deren weiblicher Identität sie nichts ahnen. Die knabenhafte Kleidung, die Asta/Hamlet (manchmal unter einem akademischen Gown) trägt, und die schicke Kurzhaarfrisur, in der sie auftritt, passen in den Trend der 1920er Jahre; der populäre Film bestimmt die garçonnehafte Jungmädchenmode dieser Zeit wesentlich mit. Insbesondere verdankt sich der deutsche Bubikopf als Zeichen der Frauenemanzipation dem Haarschnitt von Asta Nielsens Hamlet.

\footnotetext{
35 Tony Howard, Women as Hamlet (S. 142) zitiert Gade über Vining: „God knows where the man had come upon this bizarre notion, but American professors do discover the most incredible things".
} 
Jahrzehnte später, in der Zeit der zweiten Frauenbewegung, werden weibliche Hamlets noch einmal aktuell, aber sie sind jetzt frauenideologisch umstritten. Für eine feministische Kritik, die Shakespeares Stücke pauschal als Dokumente einer patriarchalischen Welt abtut, macht die Frau als Hamlet wenig Sinn. Indem eine Schauspielerin diese Rolle übernimmt, identifiziert sie sich mit den in solcher Kritik betonten frauenfeindlichen Zügen des Dramas, wie sie vor allem in Hamlets bzw. Shakespeares verächtlichem Umgang mit Ophelia und Gertrude gesehen werden. Der komplementäre Differenzfeminismus, der das Besondere, Andere des Weiblichen gegenüber dem Männlichen hervorhebt und positiv wertet, findet es ebenfalls wenig hilfreich, wenn die Frau den Mann spielt. Erst in der Zeit der dekonstruktiven Gender und Queer Studies werden weiblichen Hamlets auf der Bühne wieder eine positive - allerdings kaum noch frauenemanzipatorische Bedeutung zuerkannt. Sie belegen ganz offensichtlich den performativen Charakter der Geschlechtsidentitäten.

Mit der Geschichte der Hamlet-Rezeption durch Schriftstellerinnen und Schauspielerinnen - wie ich sie hier skizziert habe - wird lediglich eine Linie der frauenemanzipatorischen Wirkung des Shakespeareschen Werks erfasst. Die Rezeptionsgeschichten anderer Dramen könnten das Bild vervollständigen - hier wäre noch einiges zu tun. Der weibliche Anspruch auf ein gleichwertiges intellektuelles und berufliches Leben wird nicht nur durch die Identifikation mit Hamlet als Wittenberger Student erhoben, sondern deutlicher noch dadurch, dass Portia in The Merchant of Venice eine entsprechende Vorbildfunktion zuerkannt wird. Wenn sie, als Richter verkleidet, in dieser Rolle den Vertrag zwischen Antonio und Shylock so geschickt zu deuten versteht, dass eine unblutigen Lösung möglich wird, so belegt dies wiederum, dass Frauen für akademische Studien und Berufe geeignet sind, in diesem Fall für das Richteramt. Neuere Frauengeschichtsforschung hat einen Aufsatzwettbewerb von viktorianischen Schülerinnen zum Thema "My Favourite Heroine" entdeckt, in dessen Beiträgen die Mädchen mit großer Mehrheit "The Lady Lawyer" Portia im Richtertalar zu ihrer Lieblingsheldin erwählt haben. ${ }^{36}$ Für die spätviktorianische Frauenrechtlerin Lydia Becker ist, wie ihre Beiträge zum Women's Suffrage Journal belegen, Portia wiederum eine Kronzeugin für die weibliche Befähigung zum Juristenberuf. ${ }^{37}$

Andere Frauengestalten spielen in der sexuellen Emanzipation eine Rolle. So etwa Shakespeares Juliet, die ihre Liebe zu Romeo gegen konventionelle Moral-

\footnotetext{
${ }^{36}$ Dies berichtet Mark Hollingworth im Beitrag "Shakespeare Criticism“, in: Shakespeare in the Nineteenth Century, ed. Gail Marshall, Cambridge U, S. 39-59: bes. S. 41.

${ }^{37}$ Dazu Georgianna Ziegler, „Women and Shakespeare“, in: Shakespeare in the Nineteenth Century, ed. Marshall, S. 205-228.
} 
vorstellungen freizügig bekennt und ausagiert. Noch bei einem ShakespeareKongress 1992 in Tokio berichtete eine Japanerin, wie sie sich als junges Mädchen für Juliet begeistert habe und sich die Liebesdialoge von Romeo und Juliet laut vorgelesen habe - Romeos Worte in einer eigenen japanischer Übersetzung, Juliets Repliken aber auf Englisch; in ihrer Muttersprache habe sie diese noch nicht zu sprechen gewagt. Einige englische Inszenierungen des 19. Jahrhunderts versuchten, mit Rücksicht auf die jungen Mädchen im Publikum den sexuellen Zündstoff der Liebesgeschichte dadurch zu entschärfen, dass sie Romeo durch eine Frau spielen ließen. Inwieweit sie damit aus dem heterosexuellen Regen in die lesbische Traufe gerieten, wäre noch zu untersuchen,

Für viktorianische Schauspielerinnen war (weit mehr als Juliet) Shakespeares Cleopatra, die Meisterin der Liebeskünste im Römerdrama, eine Frau, deren Rolle sie begehrten. Zumeist allerdings vergeblich, denn die Theatermanager scheuten sich, eine solche Femme fatale auf die Bühne zu bringen. Es würde sich vermutlich lohnen, den oft feindseligen und angstbesetzten Kommentaren männlicher Shakespeare-Kritiker und Wissenschaftler des 19. und frühen 20. Jahrhunderts die natürlich gut erforscht sind - die weniger gut erschlossenen positiven Cleopatra-Bilder von Frauen, besonders von Schauspielerinnen, gegenüber zu stellen.

\section{Bibliographie}

Behn, Aphra, „The Dutch Lover“, The Works of Aphra Behn. The Plays 16711677, vol. 5. Hg. Janet Todd, London: Pickering, 1996.

Boutin, Aimée, „Shakespeare, Women, and French Romanticism“, Modern Language Quarterly 65,4 (2004). 505-529.

Colet, Louise. Poésies complètes, Paris: Charles Gosselin, 1844.

Droste-Hülshoff, Annette von. Briefe 1843-1848. Hg. Winfried Woesler, Tübingen: Niemeyer 1992.

Eliot, George, „A College Break-Fast Party“ (1874). Poems 1908, S. 416-440 www.unz.org/Pub/EliotGeorge-1908v09A-00001 (1.7.2014).

Goethe, Johann Wolfgang von. Wilhelm Meisters Lehrjahre. Bd. 10. Berlin, Weimar: Aufbau Verlag, 1988.

Hawley, Judith, „Shakespearean Sensibilities: Women Reading Shakespeare 17531808“, Shakespearean Continuities: Essays in Honour of E.A.J. Honigmann. Hg. Joan Batchelor u.a., Basingstoke: Macmillan, 1997. 
Howard, Tony. Women as Hamlet: Performance and Interpretation in Theatre, Film and Fiction. Cambridge: Cambridge University Press, 2007.

Jonson, Ben, „To the Memory of my Beloved, The Author Mr. William Shakespeare“, The Norton Shakespeare, Hg. Stephen Greenblatt u.a., New York: Norton, 1997.

Lewald, Fanny. Vom Sund zum Posilip: Briefe aus den Jahren 1879 bis 1881 . Paderborn: Salzwasser Verlag, 2013.

Mereau, Sophie, „Fragment eines Briefs über Wilhelm Meisters Lehrjahre“ (1799), Kalasthiskos, 1. Bd. Berlin: 1801.

Sand, George. Consuelo. Hg. Robert Sctrick, Paris: Phebus 1999.

---. „Mai 1835 an Adolphe Guéroult“, Correspondance. Hg. G. Lubin, 25 Bd., Paris: Garnier 1964-1991, Bd. III.

---. „Hamlet“, Almanach du Mois, Februar 1845. Siehe: http://www.gutenberg.org/files/28623/28623-h/28623-h.htm (1.7.2014).

Schabert, Ina, „,The Plays the Thing: George Sand's Hamlet-Novel L'Homme de Neige“, Poetica 45, 3-4 (2013) 333-346.

Taylor, Mary Scott, „The Female Advocate“, First Feminists: British Women Writers 1578-1799. Hg. Moira Ferguson, Bloomington: Indiana University Press, 1985.

Terry, Ellen. Four Lectures on Shakespeare (1911-1921), London: Martin Hopkinson, 1932.

---. The Story of my Life, New York: Schocken, 1982.

Theobald, Lewis. Shakespeare Restored (London 1726). London: The British Library, 2010. 